\title{
Non-Abelian gauge theory on noncommutative spaces
}

\author{
Peter Schupp* \\ Sektion Physik, Universität München, Theresienstr. 37, 80333 München, Germany \\ E-mail: 'schupp@theorie.physik.uni-muenchen.de'
}

ABSTRACT: We present a brief introduction to the construction of gauge theories on noncommutative spaces with star products. Particular emphasis is given to issues related to non-Abelian gauge groups and charge quantization. This talk is based on joined work with B. Jurčo, J. Madore, L. Möller, S. Schraml and J. Wess.

\section{Introduction}

The topic of this talk is the type of noncommutative gauge theory that has become a recent focus of interest in string theory, where it appears when one considers open strings in the presence of a background $B$-field [i]1] these noncommutative gauge theories as field theories, so it seems appropriate to present an intuitive approach to their construction that is independent of string theory (but closely

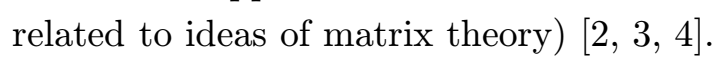

A characteristic feature of noncommutative gauge theories is the emergence of many new interactions. This includes self-couplings of gauge bosons and may even include couplings between the photon and neutral particles. One may picture these new interactions as arising from the interplay of the gauge fields and noncommutative space-time. The formalism that we shall present is particularly well-suited to capture this phenomenon. It is also the only known approach that works for arbitrary gauge groups and representations.

\section{Gauge theory on noncommutative spaces}

When trying to replace the notions and concepts of commutative geometry in the more general noncommutative framework the basic strategy is to not consider the space-time manifold itself but rather the algebra of functions on it. In the noncommutative realm this algebra is replaced by an arbitrary associative algebra. We shall refer to elements of this algebra, e.g., fields $\widehat{\Psi}, \hat{A}_{\mu}, \hat{F}_{\mu \nu}$, gauge parameters $\hat{\Lambda}$ and coordinates $x^{\mu}$, as "functions on noncommutative space-time." The simplest example of such a noncommutative space is

\footnotetext{
${ }^{*}$ Speaker.
} 
given by the canonical structure $\left[x^{\mu}, x^{\nu}\right]=i \theta^{\mu \nu}$, with a constant antisymmetric matrix $\theta^{\mu \nu}$. One could of course have more complicated structures, e.g., with commutation relations that close linearly (Lie structure) or quadratically (quantum space structure). A priori there is no reason to expect that $\theta^{\mu \nu}$ is constant, but we shall concentrate on that case in the following for simplicity of presentation. We use the symbol $\star$ to denote the product of the noncommutative structure; this does not need to be a star product, but we are here especially interested in noncommutative structures that have a well-defined classical limit and lend themselves to a perturbative formulation, as is the case for star products.

The construction of a gauge theory on a given non-commutative space can be based on a few fundamental ideas: the concept of covariant coordinates, the requirement of locality, gauge equivalence and consistency conditions.

\subsection{Covariant coordinates}

The infinitesimal non-commutative gauge transformation of a fundamental matter field is

$$
\hat{\delta} \widehat{\Psi}=i \hat{\Lambda} \star \widehat{\Psi} .
$$

In the non-Abelian case the symbol $\star$ includes also matrix multiplication. We observe that multiplying $\widehat{\Psi}$ on the left by a coordinate $x^{\mu}$ is not a covariant operation because the gauge parameter does not commute with it:

$$
\hat{\delta}\left(x^{\mu} \star \widehat{\Psi}\right)=i x^{\mu} \star \hat{\Lambda} \star \widehat{\Psi} \neq i \hat{\Lambda} \star x^{\mu} \star \widehat{\Psi} .
$$

In complete analogy to the covariant derivatives of ordinary gauge theory we need to introduce covariant coordinates $X^{\mu}=x^{\mu}+\hat{A}^{\mu}$ where $\hat{A}^{\mu}$ is a non-commutative analog of the gauge potential. In the case of constant, non-degenerate $\theta^{\mu \nu}$ it is more convenient to work with $\hat{A}_{\nu}$, where $\hat{A}^{\mu}=\theta^{\mu \nu} \hat{A}_{\nu}$, with

$$
\hat{\delta} \hat{A}_{\mu}=\partial_{\mu} \hat{\Lambda}+i\left[\hat{\Lambda} \stackrel{\star}{,} \hat{A}_{\mu}\right] .
$$

Similarly, covariant functions $\mathcal{D}(f)=f+\mathcal{A}(f)$ can be introduced [i coordinates one can construct further covariant objects including the non-commutative field strength

$$
\widehat{F}_{\mu \nu}=\partial_{\mu} \hat{A}_{\nu}-\partial_{\nu} \hat{A}_{\mu}-i\left[\hat{A}_{\mu} \stackrel{\star}{\hat{A}_{\nu}}\right], \quad \hat{\delta} \widehat{F}_{\mu \nu}=i\left[\hat{\Lambda} \stackrel{\star}{,} \widehat{F}_{\mu \nu}\right]
$$

related to the commutator of covariant coordinates, and the covariant derivative

$$
\widehat{D}_{\mu} \widehat{\Psi}=\partial_{\mu} \widehat{\Psi}-i \hat{A}_{\mu} \star \widehat{\Psi}
$$

related to the covariant expression $X^{\mu} \star \widehat{\Psi}-\widehat{\Psi} \star x^{\mu}$.

\subsection{Classical limit and locality}

A star product of two functions $f, g$ is a formal power series starting with the ordinary product plus higher order terms that are chosen so as to yield an associative product. The star product can be pictured as a tower build upon the leading Poisson tensor $\theta^{\mu \nu}$ :

$$
f \star g=f \cdot g+\frac{i}{2} \theta^{\mu \nu} \partial_{\mu} g \cdot \partial_{\nu} f+\ldots
$$


It is a natural to ask whether it is possible to express also the non-commutative fields $\hat{A}, \widehat{\Psi}$ and non-commutative gauge parameter $\hat{\Lambda}$ in a similar fashion as towers build upon the corresponding ordinary fields $A, \Psi$ and ordinary gauge parameter $\Lambda$. This is indeed the case; there are so-called Seiberg-Witten maps [i] $\left[\begin{array}{l}1 \\ 1\end{array}\right]$ that express the non-commutative quantities as local functions of the ordinary fields:

$$
\begin{aligned}
\hat{A}_{\mu} & =A_{\mu}+\frac{1}{4} \theta^{\xi \nu}\left(\left\{A_{\nu}, \partial_{\xi} A_{\mu}\right\}+\left\{F_{\xi \mu}, A_{\nu}\right\}\right)+\ldots \\
\widehat{\Psi} & =\Psi+\frac{1}{2} \theta^{\mu \nu} A_{\nu} \partial_{\mu} \Psi+\frac{i}{8} \theta^{\mu \nu}\left[A_{\mu}, A_{\nu}\right] \Psi+\ldots \\
\hat{\Lambda} & =\Lambda+\frac{1}{4} \theta^{\mu \nu}\left\{A_{\nu}, \partial_{\mu} \Lambda\right\}+\ldots
\end{aligned}
$$

where $F_{\mu \nu}=\partial_{\mu} A_{\nu}-\partial_{\nu} A_{\mu}-i\left[A_{\mu}, A_{\nu}\right]$ is the ordinary field strength. By a local function of a field we mean a formal series that at each order in $\theta$ depends on the field and a finite number of derivatives of the field. We shall henceforth use a hat ${ }^{\wedge}$ to denote quantities that are understood to be expanded as local functions of their classical counterparts via Seiberg-Witten maps.

\subsection{Gauge equivalence and consistency condition}

The Seiberg-Witten maps $\left(\overline{2} \cdot \overline{6}_{i}\right)-\left(\overline{2}-\overline{2}_{1}^{\prime}\right)$ have the remarkable property that ordinary gauge transformations $\delta_{\Lambda} A_{\mu}=\partial_{\mu} \Lambda+i\left[\Lambda, A_{\mu}\right]$ and $\delta_{\Lambda} \Psi=i \Lambda \cdot \Psi$ induce noncommutative gauge transformations $\left(\overline{2} \cdot \overline{1}_{1}^{\prime}\right),(\overline{2} \cdot \overline{2})$ of $\hat{A}, \widehat{\Psi}, \hat{\Lambda}$. Furthermore, any pair of non-commutative gauge parameters $\hat{\Lambda}, \widehat{\Sigma}$ has to satisfies the following consistency condition [4]

$$
[\hat{\Lambda} \star \widehat{\Sigma}]+i \delta_{\Lambda} \widehat{\Sigma}-i \delta_{\Sigma} \hat{\Lambda}=\widehat{[\Lambda, \Sigma]}
$$

The gauge equivalence and consistency conditions do not uniquely determine SeibergWitten maps. To the order considered here we have the freedom of classical field redefinitions and noncommutative gauge transformations. We have used that freedom to choose maps with Hermitean $\hat{A}_{\mu}$ and $\hat{\Lambda}$. Other choices are possible. A particularly simple set of maps can, e.g., be obtained from $(\overline{2} .6)-(\overline{2} \cdot \overline{2} . \overline{8})$ by a noncommutative gauge transformation generated by $\frac{1}{4} \theta^{\mu \nu} A_{\nu} A_{\mu}$.

The freedom in the Seiberg-Witten map is essential for the renormalization of noncommutative gauge theory [i, i $]$. It is also important in the context of tensor products of gauge groups. For instance, for a field $\Phi$ that transforms on the left and on the right under two arbitrary gauge groups, we have the following hybrid Seiberg-Witten map,

$$
\widehat{\Phi}=\Phi+\frac{1}{2} \theta^{\mu \nu}\left[A_{\nu}\left(\partial_{\mu} \Phi-\frac{i}{2}\left(A_{\mu} \Phi-\Phi A_{\mu}^{\prime}\right)\right)+\left(\partial_{\mu} \Phi-\frac{i}{2}\left(A_{\mu} \Phi-\Phi A_{\mu}^{\prime}\right)\right) A_{\nu}^{\prime}\right]+\ldots
$$

Under $\delta \Phi=i \Lambda \Phi-i \Phi \Lambda^{\prime}, \delta A_{\nu}=\partial_{\nu} \Lambda+i\left[\Lambda, A_{\nu}\right], \delta A_{\nu}^{\prime}=\partial_{\nu} \Lambda^{\prime}+i\left[\Lambda^{\prime}, A_{\nu}^{\prime}\right]$ we find

$$
\delta \widehat{\Phi}=i \widehat{\Lambda} \star \widehat{\Phi}-i \widehat{\Phi} \star \widehat{\Lambda}^{\prime}
$$




\section{Non-Abelian gauge groups in noncommutative setting}

The commutator of two Lie algebra-valued noncommutative gauge parameters,

$$
\left[\hat{\Lambda} \stackrel{\star}{,} \hat{\Lambda}^{\prime}\right]=\frac{1}{2}\left\{\Lambda_{a}(x) \stackrel{\star}{,} \Lambda_{b}^{\prime}(x)\right\}\left[T^{a}, T^{b}\right]+\frac{1}{2}\left[\Lambda_{a}(x) \stackrel{\star}{,} \Lambda_{b}^{\prime}(x)\right]\left\{T^{a}, T^{b}\right\},
$$

does not close in the Lie algebra, because the coefficient of $\left\{T^{a}, T^{b}\right\}$ is in general non-zero. (The only important exception is $U(N)$ in the fundamental representation.) We thus have to consider enveloping algebra-valued noncommutative gauge parameters

$$
\hat{\Lambda}=\Lambda_{a}^{0}(x) T^{a}+\Lambda_{a b}^{1}(x): T^{a} T^{b}:+\Lambda_{a b c}^{2}(x): T^{a} T^{b} T^{c}:+\ldots,
$$

and fields $\hat{A}_{\mu}[\overline{2}]$. A priori, it appears that we then have an infinite number of degrees of freedom. Via the Seiberg-Witten map, however, all the terms in $\hat{\Lambda}$ and $\hat{A}_{\mu}$ can be expressed in terms of a finite number of classical parameters and fields.

\section{Noncommutative Yang-Mills action}

For constant $\theta$ the ordinary integral is a trace for the $\star$-product: $\int f \star g=\int g \star f=\int f g$. An invariant action for the gauge potential and the matter fields is

$$
S=\int d^{4} x\left[-\frac{1}{2 g^{2}} \operatorname{tr} \widehat{F}_{\mu \nu} \star \widehat{F}^{\mu \nu}+\overline{\widetilde{\Psi}} \star\left(i \gamma^{\mu} \widehat{D}_{\mu}-m\right) \widehat{\Psi}\right],
$$

where $\widehat{D}_{\mu} \widehat{\Psi} \equiv \partial_{\mu} \widehat{\Psi}-i \hat{A}_{\mu} \star \widehat{\Psi}, \widehat{F}_{\mu \nu}=\partial_{\mu} \hat{A}_{\nu}-\partial_{\nu} \hat{A}_{\mu}-i\left[\hat{A}_{\mu} \star \hat{A}_{\nu}\right]$. Expanding $\hat{A}_{\mu}$ and $\widehat{\Psi}$ to first order in $\theta$ using the Seiberg-Witten maps ( $\left(\overline{2} \cdot \overline{6}_{1}^{*}\right),\left(\overline{2} \cdot \overline{2}_{1}\right)$ yields

$$
\begin{aligned}
S= & \int d^{4} x\left[-\frac{1}{2 g^{2}} \operatorname{tr} F_{\mu \nu} F^{\mu \nu}+\frac{1}{4 g^{2}} \theta^{\mu \nu} \operatorname{tr} F_{\mu \nu} F_{\rho \sigma} F^{\rho \sigma}-\frac{1}{g^{2}} \theta^{\mu \nu} \operatorname{tr} F_{\mu \rho} F_{\nu \sigma} F^{\nu \sigma}\right. \\
& \left.+\bar{\psi}(i \not D-m) \psi-\frac{1}{4} \theta^{\mu \nu} \bar{\psi} F_{\mu \nu}(i \not D-m) \psi-\frac{i}{2} \theta^{\mu \nu} \bar{\psi} \gamma^{\rho} F_{\rho \mu} D_{\nu} \psi\right]
\end{aligned}
$$

with $D_{\mu} \psi \equiv \partial_{\mu} \psi-i A_{\mu} \psi$ and $F_{\mu \nu}=\partial_{\mu} A_{\nu}-\partial_{\nu} A_{\mu}-i\left[A_{\mu}, A_{\nu}\right]$.

\section{Charge in noncommutative QED}

The only couplings in addition to (2.2. field $\widehat{\Psi}$ compatible with the non-commutative gauge transformation $(\overline{2} 2.2 i)$ are $\partial_{\mu} \widehat{\Psi}-i \widehat{\Psi} \star \hat{A}_{\mu}$ and $\partial_{\mu} \widehat{\Psi}+i\left[\hat{A}_{\mu} \stackrel{\star}{\Psi}\right]$. It thus appears that only $\mathrm{U}(1)$ charges $1,-1,0$ are possible. (The latter possibility shows how a neutral particle can couple to an abelian gauge field in a noncommutative setting.) We should of course consider physical fields $\hat{a}_{\mu}^{(n)}(x)$. Let $Q$ be the generator of $U(1)$ (charge operator), $e$ a coupling constant and $\psi^{(n)}$ a field for a particle of charge $q^{(n)}$. Then $A_{\mu}=e Q a_{\mu}(x)$ and $\hat{A}_{\mu} \star \hat{\psi}^{(n)}=e q^{(n)} \hat{a}_{\mu}^{(n)}(x) \star \hat{\psi}^{(n)}$. The Seiberg-Witten map $\hat{A}_{\mu}$ depends explicitly on $Q$. In ordinary QED there is only one photon, i.e., there is no need for a label $(n)$ on $a_{\mu}$. Here, however, we have a separate $\hat{a}_{\mu}^{(n)}$ for every charge $q^{(n)}$ in the theory, because due to the $\star$-commutator in the transformation of $\hat{a}_{\mu}^{(n)}$ it is not possible to absorb $q^{(n)}$ in a redefinition of $\hat{a}_{\mu}^{(n)}$. We can have any charge now, but it appears that we have too many degrees of freedom. This is not the case, however, since the $\hat{a}_{\mu}^{(n)}$ are not independent. They are local functions of the correct number of classical gauge fields

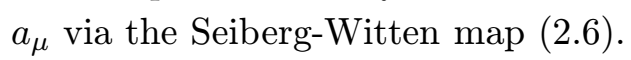




\section{Construction of the Seiberg-Witten map}

In the Abelian case the Seiberg-Witten map is known explicitly for any Poisson structure $\theta(x)$ and corresponding Kontsevich $\star$-product $[\overline{5}]$. The construction is based on equivalent star products $\star, \star^{\prime}$ that are quantizations of Poisson structures $\theta$ and $\theta^{\prime}=\theta(1+F \theta)^{-1}$. There is also a path-integral formulation based on noncommutative Wilson lines [i]. The non-Abelian case is technically more involved and only formally related to the Abelian case. For a cohomological approach based on the consistency relation (2.29.9.

\section{Finite gauge transformations and noncommutative vector bundle}

The infinitesimal gauge parameter $\hat{\Lambda} \equiv \hat{\Lambda}_{[A]}$ can be promoted to a full finite noncommutative gauge transformation $\widehat{G}_{[A]}=\exp _{\star}\left(\delta_{\Lambda}\right) \star \exp _{\star}\left(-\delta_{\Lambda}+i \hat{\Lambda}_{[A]}\right)$ corresponding to a group element $G=e^{i \Lambda}$. The consistency relation (2.9. law" [ịi]

$$
{\widehat{G_{1}\left[A_{G_{2}}\right]}} \star \widehat{G}_{2[A]}={\widehat{G_{1} \cdot G_{2}}}_{[A]},
$$

with the gauge transformed gauge potential $A_{G_{2}}$ in the first factor. The $\hat{G}_{[A]}$ can be used as transition functions in the construction of noncommutative vector bundles, which are the underlying mathematical structure of the noncommutative gauge theories that we have been considering [is].

\section{Acknowledgements}

I would like to thank B. Jurčo, J. Madore, L. Möller, S. Schraml and J. Wess for fruitful collaboration and P. Aschieri and W. Behr for helpful discussions.

\section{References}

[1] N. Seiberg, E. Witten, JHEP 9909, 032 (1999) [hep-th/9908142].

[2] J. Madore, S. Schraml, P. Schupp, J. Wess, Eur. Phys. J. C 16, 161 (2000) [hep-th/0001203]; B. Jurčo, S. Schraml, P. Schupp, J. Wess, Eur. Phys. J. C 17, 521 (2000) [hep-th/0006246].

[3] A. A. Bichl, J. M. Grimstrup, L. Popp, M. Schweda, R. Wulkenhaar, hep-th/0102103.

[4] B. Jurčo, L. Möller, S. Schraml, P. Schupp, J. Wess, Eur. Phys. J. C 21, 383 (2001) [hep-th/0104153].

[5] B. Jurčo, P. Schupp, Eur. Phys. J. C 14, 367 (2000) [hep-th/0001032];

B. Jurčo, P. Schupp, J. Wess, Nucl. Phys. B 584, 784 (2000) [hep-th/0005005];

B. Jurčo, P. Schupp, J. Wess, Nucl. Phys. B 604, 148 (2001) [hep-th/0102129].

[6] A. Bichl, J. Grimstrup, H. Grosse, L. Popp, M. Schweda, R. Wulkenhaar, JHEP 0106, 013 (2001) [hep-th/0104097].

[7] K. Okuyama, JHEP 0003, 016 (2000) [hep-th/9910138].

[8] D. Brace, B. L. Cerchiai, A. F. Pasqua, U. Varadarajan, B. Zumino, JHEP 0106, 047 (2001) [hep-th/0105192]; D. Brace, B. L. Cerchiai, B. Zumino, hep-th/0107225.

[9] B. Jurčo, P. Schupp, J. Wess, hep-th/0106110. 